\title{
Targeting Cre Recombinase to Specific Neuron Populations with Bacterial Artificial Chromosome Constructs
}

\author{
Shiaoching Gong, ${ }^{1}$ Martin Doughty, ${ }^{1}$ Carroll R. Harbaugh, ${ }^{4}$ Alexander Cummins, ${ }^{4}$ Mary E. Hatten, ${ }^{2}$ Nathaniel Heintz, ${ }^{3}$ \\ and Charles R. Gerfen ${ }^{4}$ \\ ${ }^{1}$ Gene Expression Nervous System Atlas Project, ${ }^{2}$ Laboratory of Developmental Neurobiology, and ${ }^{3}$ Laboratory of Molecular Biology, Howard Hughes \\ Medical Institute, The Rockefeller University, New York, New York 10021, and ${ }^{4}$ Laboratory of Systems Neuroscience, National Institute of Mental Health, \\ Bethesda, Maryland 20892
}

Transgenic mouse lines are characterized with Cre recombinase driven by promoters of CNS-specific genes using bacterial artificial chromosome (BAC) constructs. BAC-Cre constructs for 10 genes (Chat, Th, Slc6a4, Slc6a2, Etv1, Ntsr1, Drd2, Drd1, Pcp2, and Cmtm5) produced 14 lines with Cre expression in specific neuronal and glial populations in the brain. These Cre driver lines add functional utility to the $>500$ BAC-EGFP (enhanced green fluorescent protein) transgenic mouse lines that are part of the Gene Expression Nervous System Atlas Project.

\begin{abstract}
Introduction
The ability to target genetic manipulations to specific CNS cell types is essential for analysis of genes, cells, and circuits that play key roles in behavior and disease. Limiting genetic manipulations to specific structures, cell types, or developmental periods can overcome many of the problems encountered using traditional genetargeting approaches (Lakso et al., 1992; Gu et al., 1994; Kuhn et al., 1995; Branda and Dymecki, 2004). These include complications resulting from early embryonic lethality or peripheral phenotypes for widely expressed genes, the interpretation
\end{abstract}

\footnotetext{
Received March 29, 2007; accepted July 10, 2007.

The Gene Expression Nervous System Atlas Project was supported by National Institute of Neurologic Disease and Stroke Grants N01 NS-0-2331 and N01 NS-7-2370, the National Institute of Mental Health (NIMH), and the National Institutes of Health Neuroscience Blueprint. C.R.G. was supported by the Intramural Research Program, NIMH. S.G. and N.H. were supported by the Simons Foundation. N.H. is an investigator of the Howard Hughes Medical Institute. We are grateful for the excellent technical support of Cuidong Wang and Sanjay Mehta.

Correspondence should be addressed to Charles R. Gerfen, Building 111, National Institutes of Health Animal Center, 16701 Elmer School Road, Dickerson, MD 20842. E-mail: gerfenc@mail.nih.gov.

DOI:10.1523/JNEUROSCI.2707-07.2007

Copyright $\odot$ 2007 Society for Neuroscience $\quad$ 0270-6474/07/279817-07\$15.00/0
}

of gross developmental phenotypes resulting from mutation of critical CNSexpressed genes, and the investigation of complex behavioral phenotypes that result from disruptions of broadly expressed genes. For example, one of the main advantages of the use of mouse models for human neurologic diseases is the potential to limit genetic manipulations to specific times, structures, or cell types, thus providing an avenue for dissection of the complex mechanisms of disease. The most common technique for genetic analysis in specific cell types involves cellspecific expression of Cre or Flp recombinases crossed into mice carrying designer alleles of target genes to mutate those genes only in those cells expressing the appropriate recombinase (Kuhn and Torres, 2002; Branda and Dymecki, 2004). However, a large variety of other types of experimentation, such as lineage tracing or circuit-mapping studies, can also be achieved using variations of this strategy (Zinyk et al., 1998; Soriano, 1999; Braz et al., 2002). All of these applications depend on the expression of functionally relevant concentrations of the appropriate recom- binase in specific cell types or structures in vivo. Some transgenic lines have been produced with targeted neuronal Cre expression, including catecholamine, dopamine, serotonin, and specific cerebellar or cortical neuron populations (Tsien et al., 1996; Aller et al., 2003; Lindeberg et al., 2004; Scott et al., 2005; Zhuang et al., 2005). Although such lines demonstrate the utility of this powerful genetic strategy, the current number of lines is relatively limited compared with the large diversity of CNS cell types.

The use of bacterial artificial chromosome (BAC) vectors in transgenic mice is one strategy to achieve efficient and reproducible cell-specific expression of proteins of interest in vivo (Yang et al., 1997; Heintz, 2001; Gong et al., 2002). The efficacy of this approach for analysis of specific CNS-expressed genes has recently been demonstrated in the Gene Expression Nervous System Atlas (GENSAT) project, which has used engineered BAC vectors to target enhanced green fluorescent protein (EGFP) expression to specific CNS cell types (Gong et al., 2003). The ability of a given BAC vector to drive cor- 
rect expression in transgenic mice is a result of their ability to accommodate dispersed regulatory sequences across relatively large regions of the genome (tens to hundreds of kilobases) for a given gene, such that reproducible expression from BAC vectors has been achieved for $>500$ genes so far analyzed in the GENSAT project (www.gensat.org; www. ncbi.nlm.nih.gov/projects/gensat). Thus, the BAC vectors identified by GENSAT provide a rich potential resource for the creation of recombinase driver lines that target cell types evident in the GENSAT database.

\section{BAC transgene construction and characterization of Cre expression}

To determine whether engineered BACs can be used generally to efficiently drive functional levels of constitutive or inducible Cre recombinases in specific CNS cell types, we have chosen to use 10 different $\mathrm{BAC}$ vectors to create transgenic lines expressing recombinase (Table 1). For the purpose of this study, BACs were selected from those previously characterized with BAC-EGFP constructs from the GENSAT project. Genes selected for this study represented those expressed selectively in neurotransmitter-specific populations, in specific components of forebrain neural circuits, or in specific neuron or glial populations. In each case, BAC engineering (Gong et al., 2002) was used to insert an intron containing Cre or CreERT2 cassettes, followed by a polyadenylation sequence to terminate transcription of the fusion transcript immediately after the recombinase gene, into the BAC vector at the initiating ATG codon in the first coding exon of the gene chosen for expression. This was done using a new shuttle vector (pLD53.SC-Cre) carrying the appropriate selectable markers and a small homology arm from the locus being targeted to guide recombination. An inducible recombinase driver line using CreERT2 (Indra et al., 1999) was also analyzed. Offspring of founders were crossed to a Rosa26/stop/EGFP reporter line (Soriano, 1999). Functional recombination of the reporter locus was analyzed for all lines using immunohistochemical detection of EGFP in adult mice carrying both the BAC construct and Rosa26/ EGFP transgene. Because copy number, transgene insertion sites, and transient developmental expression of the chosen loci in previously undetected cell types could result in unexpected sites of recombinase activity, multiple transgenic founder lines for each gene were generated and ana-

Table 1. List of BAC-Cre lines described in this study

\begin{tabular}{|c|c|c|}
\hline Gene [BAC-Cre transgenic line(s)] & BAC clone & Cre expression pattern (adult/developmental/partial) \\
\hline Choline acetyl transferase (Chat) & RP23-246B12 & \\
\hline GM24/GM60 & & Adult \\
\hline GM53 & & Partial: motor nuclei only \\
\hline Tyrosine hydroxylase (Th) & RP23-350E13 & \\
\hline $\mathrm{F} \mid 12 / \mathrm{Fl} 172$ & & Adult plus developmental pattern \\
\hline Serotonin transporter (Slca4) & RP24-335M24 & \\
\hline ET33/ET35 & & Adult \\
\hline ET124 & & Adult plus developmental pattern \\
\hline Noradrenalin transporter (SIc6a2) & RP23-109023 & \\
\hline FV317/FV319 & & Adult \\
\hline ETS domain transcription factor (Etv1) & RP23-250K4 & \\
\hline GM225 & & Neocortex layer 5 \\
\hline Neurotensin receptor 1 (Ntsr1) & RP23-314D14 & \\
\hline GN220 & & Neocortex layer 6 \\
\hline $\mathrm{D}_{2}$ dopamine receptor (Drd2) & RP23-161H15 & \\
\hline ER44 & & Adult \\
\hline ER43 & & Partial: striatopallidal neurons \\
\hline $\mathrm{D}_{1}$ dopamine receptor $(\operatorname{Drd} 1 \mathrm{a})$ & $\mathrm{RP} 23-47 \mathrm{M} 2$ & \\
\hline EY262/FK161/FK150 & & Adult \\
\hline Purkinje cell protein (Pcp2) & RP24-186D18 & \\
\hline GN135 & & Adult \\
\hline Oligodendrocyte protein ( $\mathrm{Cmtm5}$ ) & RP24-317F19 & \\
\hline GM160 & & Adult \\
\hline
\end{tabular}

lyzed. We report here that generation of Cre driver lines that express functional Cre in the expected cell types was achieved for all 10 engineered BAC vectors tested, including one inducible with tamoxifen. As predicted, in some cases there were variations in the patterns of Cre-induced EGFP expression, which are instructive in the general use of BACs to direct Cre expression in targeted neuronal and glial cell types. The patterns of functional Cre expression for 14 BAC-Cre transgenic lines are described.

\section{Neurotransmitter-specific genes}

A mainstay of neuroscience research involves pharmacologic manipulation of specific neurotransmitter systems. Targeting genetic manipulations to neurons based on their neurotransmitter phenotype greatly expands approaches for manipulating such systems. For this purpose, four BAC-Cre constructs were used, which included two genes encoding neurotransmitter-synthesizing enzymes, tyrosine hydroxylase (Th) and choline acetyl transferase (Chat), and two genes encoding neurotransmitter transporters, the serotonin transporter (Slc6a4) and the noradrenalin transporter (Slc6a2). For two of these genes, Th and Slca4, Cre driver lines using knock-in approaches have been produced (Lindeberg et al., 2004; Zhuang et al., 2005), which allows for a comparison with the BAC-Cre constructs used here. For each gene, transgenic lines were produced with Cre specif- ically expressed in the appropriate adult neurotransmitter-specific neuron population as verified with double labeling for a protein marker specific for each neuron population (Fig. 1). In a Th BAC-Cre line (FI12), functional Cre expression is present in all of the catecholamine dopaminergic and noradrenergic neurons that express $T h$ in the adult (for example, in the dopaminergic neurons in the substantia nigra pars compacta) (Fig. $1 A$ ). In a Slc6a4 BAC-Cre transgenic line (ET33), functional Cre is demonstrated in serotonin neurons in the dorsal and median raphe nuclei by colocalization of tryptophan hydroxylase immunoreactivity (IR) (Fig. 1B). Acetyl choline neurons display functional Cre in a Chat BAC-Cre line (GM24) as demonstrated by colocalization of Chat immunoreactivity in neurons in the basal forebrain (see Fig. 3A).

A detailed mapping of Cre-induced EGFP in the brain for each of these transgenic lines is provided on the GENSAT website (http://www.gensat.org/index. html), and the distributions match the expression of the genes in the adult brain, for the most part. Variations in these lines and in other lines produced with the same BAC-Cre constructs for these genes are instructive. The two main types of variations encountered are those producing ectopic expression or partial expression. Ectopic expression, in which Cre-induced Rosa26 EGFP is observed in neurons that do not express the endogenous gene in the adult brain, was observed in the Th BAC- 

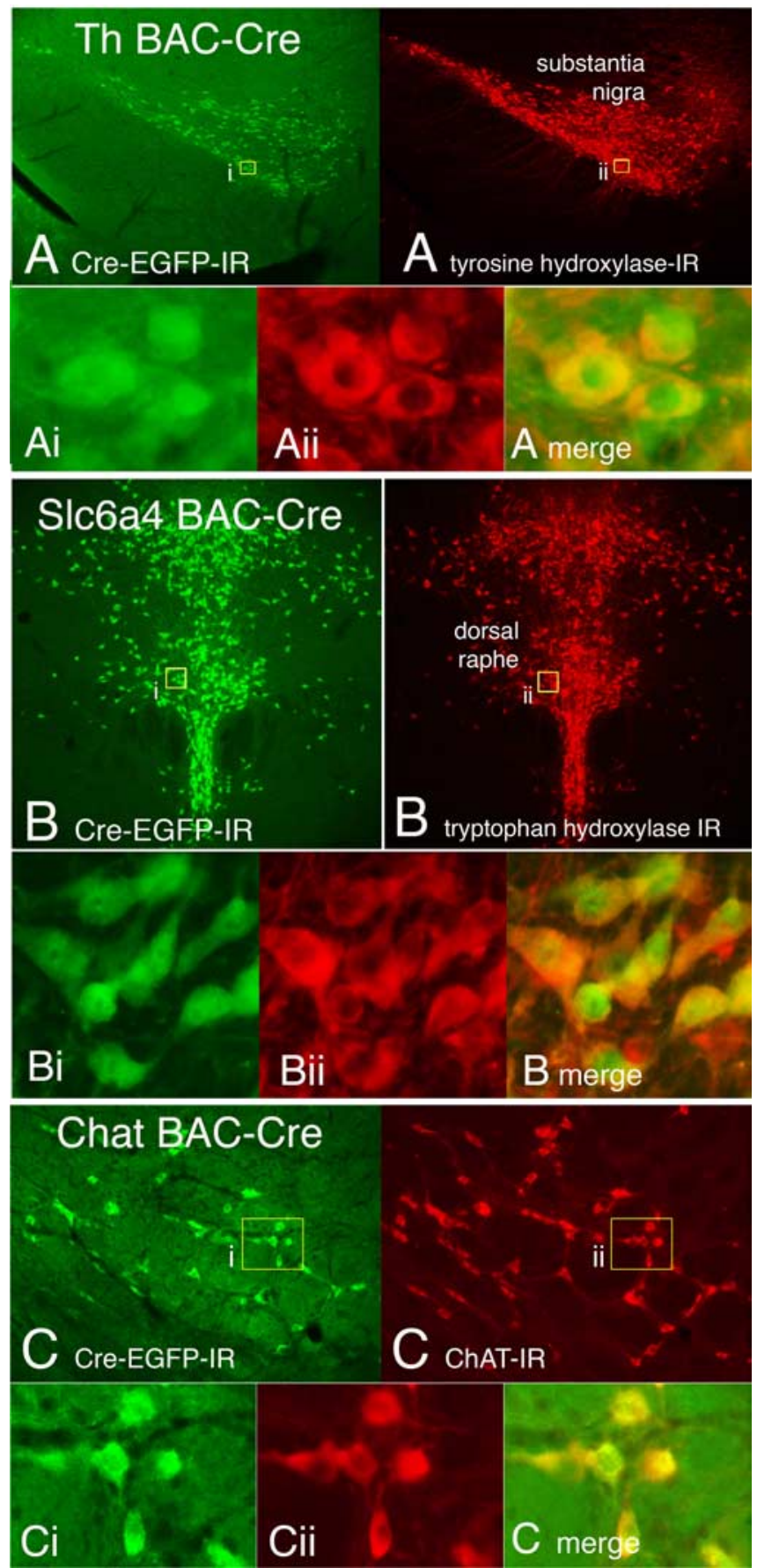

Figure 1. Cre expression directed to dopaminergic $(\boldsymbol{A})$, serotonergic $(\boldsymbol{B})$, and cholinergic $(\boldsymbol{C})$ neurons in BAC-Cre transgenic mouse lines. $A$, Dopaminergic neurons are labeled in a Th BAC-Cre transgenic line with Cre expression localized in neurons in the midbrain substantia nigra pars compacta (Th BAC-CRE; green), which colocalize with Th-IR. There is a nearly complete overlap of TH Cre-driven EGFP labeling and Th-IR (insets). $\boldsymbol{B}$, In a serotonin transporter (SIca4) BAC-Cre transgenic line, Cre is directed to serotonergic neurons in the dorsal raphe nucleus in the dorsal midbrain, which coexpress serotonin-synthesizing enzyme, tryptophan hydroxylase-IR. Insets, There is a nearly complete overlap of SIca4 Cre-driven EGFP labeling and tryptophan hydroxylase-IR in neurons in the dorsal raphe. $\boldsymbol{C}$, In a Chat BAC-Cre transgenic line, labeled neurons in the basal forebrain coexpress Chat immunoreactivity (ChAT-IR).

Cre lines and in some but not all of the Slc6a4 BAC-Cre lines. In two Th BAC-Cre lines (FI12 and FI172), Cre-induced EGFP expression is present in essentially all adult neurons in which Th-IR is present. Additionally, EGFP is also observed in selected neurons that are Th-IR its expression and transient expression of the gene in neurons during development, which do not express the gene in the adult. In this case, several reasons point to the latter mechanism. First, multiple studies have demonstrated transient developmental expression of Th in the same forebrain areas seen in the Th BAC-Cre lines (Komori et al., 1991; Marin et al., 2005). Second, other transgenic Th-Cre lines produced with different transgene vectors using a knock-in approach produce patterns of ectopic Cre expression nearly identical to those seen in the Th BAC-Cre line, which were ascribed to transient developmental expression (Lindeberg et al., 2004; Zhuang et al., 2005). Moreover, the multiple Th BAC-Cre lines analyzed in this study produce identical ectopic expression patterns. Because Th BAC-Cre constructs likely insert into different loci in different lines, it is unlikely that different insertional loci would produce identical patterns of ectopic expression (Heintz, 2001). Similar variations of Cre expression were obtained with the Slca4 BACCre construct. In two lines (ET33 and ET35), Cre expression was localized selectively to adult neurons using serotonin as a neurotransmitter, most notably serotonin neurons in the dorsal and median raphe (Fig. $1 B$ ). Another line (ET124) generated with this BAC-Cre construct displayed expression not only in adult serotonin neurons, but also in corticothalamic and thalamocortical neurons (supplemental Fig. 3, available at www. jneurosci.org as supplemental material). These latter neurons have been shown to express the serotonin transporter transiently during development (Lebrand et al., 1998) and have shown Cre expression using other Cre constructs (Zhuang et al., 2005). Thus, in this line, ectopic Creinduced EGFP expression is likely caused by transient expression of the Slca 4 gene during development. In the case of the ectopic Cre-induced EGFP expression seen in both the Th and Slca4 BAC-Cre lines, the pattern of expression is very specific and restricted to neurons expressing the endogenous genes transiently during development.

The second type of variation produces partial patterns of Cre-induced EGFP expression compared with the expression of the endogenous gene. Such a partial pattern of expression is produced in one of the Chat BAC-Cre transgenic lines. Two Chat BAC-Cre transgenic lines (GM23 and GM54) produced Cre-induced EGFP expression throughout the brain and spinal cord that matches the expression of 
Chat in the adult. Another Chat BAC-Cre line (GM60) produced Cre-induced EGFP expression, which is restricted only to motor neurons in the brainstem and spinal cord (supplemental Fig. 4, available at www.jneurosci.org as supplemental material). In this case, the more restricted expression is most likely caused by a reduced number of Chat BAC-Cre construct copies being incorporated into the genome compared with the line in which Cre is expressed in the full complement of cholinergic neurons in the brain. Evidence for this mechanism is somewhat empirical, based on the robustness of Creinduced EGFP expression in the Chat BAC-Cre line labeling all cholinergic neurons. In these lines, EGFP labeling of forebrain neurons reveals only cell bodies and dendrites, whereas motor neurons are more robustly labeled, with axonal projections clearly labeled. This suggests that there are relative differences between forebrain cholinergic neurons and motor neurons in the level of expression of Chat. Thus, differences in Cre-induced EGFP expression between these lines may reflect the level of Cre expression, which is affected by the number of copies of the construct incorporated in the different lines.

Variations of Cre expression patterns produced with BAC-Cre drivers to target neurotransmitter-specific populations are instructive. On the one hand, Credriver lines have been produced with expression restricted to the target neuron populations in the adult. On the other hand, the variations in patterns produced with some lines demonstrate the need to carefully characterize the patterns of expression. In the case of ectopic expression caused by transient gene expression during development, there are several points to be made. First, such cases underscore the necessity of using a reporter line such as the Rosa26 EGFP line to characterize Cre driver lines. Such variations may produce greater specificity of Cre expression or may require use of other BAC-Cre driver lines to more specifically target particular neuron populations.

\section{Genes expressed in specific forebrain circuits}

Whereas targeting neuron populations based on neurotransmitter phenotype is useful for certain brain systems, other systems require a different strategy. Neural circuits in the forebrain provide such a case. For example, there are a large variety of subtypes of principal projection neurons in the cerebral cortex with distinct patterns of connections, which are orga-
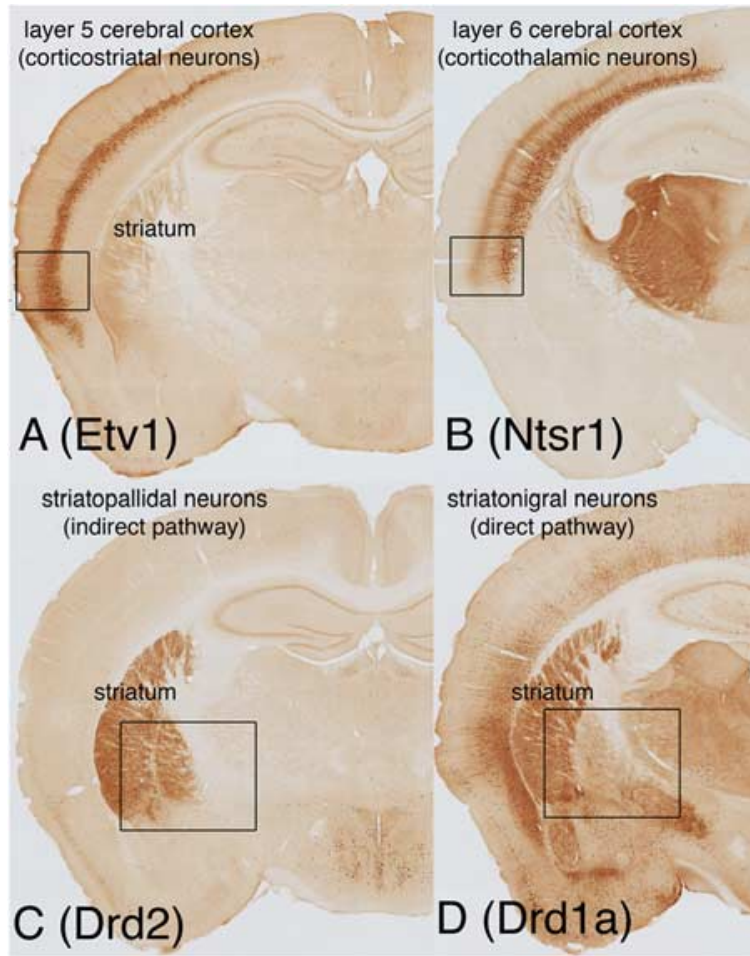

B (Ntsr1)

striatonigral neurons (direct pathway)

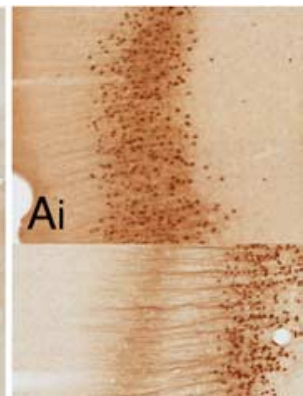

Figure 2. Cre expression in forebrain circuits, with labeling of specific neuronal projection systems in the cerebral cortex $(\boldsymbol{A}$, Etv1; $\boldsymbol{B}$, Ntsr1) and striatum (C,Drd2; $\boldsymbol{D}, \operatorname{Drd1a})$. Expression is produced in layer 5 corticostriatal neurons in an Etv1 BAC-Cre line $(\boldsymbol{A}$, Etv1). Cre is expressed in layer 6 corticothalamic neurons in an ntsr1 BAC-Cre line ( $\boldsymbol{B}, N t s r 1)$. Clearly labeled are the projection axons of these neurons, which terminate in the dorsal thalamic nuclei. In the striatum, the majority of neurons are medium spiny projection neurons, which are evenly divided into striatopallidal (indirect pathway) and striatonigral (direct pathway) neurons, which selectively express the $\mathrm{drd} 2$ and drd1a dopamine receptors, respectively. Cre expression produced in Drd2 BAC-Cre lines ( $\boldsymbol{C}$, Drd2) is directed to striatopallidal neurons. In this line, labeled neurons in the striatum extend axons that terminate in the globus pallidus external segment (GPe). In contrast, expression produced in Drd1a BAC-Cre lines ( D, Drd1a) is directed to striatonigral neurons, which have axons that extend through the globus pallidus to terminate in the internal segment of the globus pallidus (GPi) and substantia nigra (not shown).

nized into layers and cytoarchitecturally defined areas. Because all of these use glutamate as a neurotransmitter, targeting cortical neuron subtypes requires the selection of genes unrelated to their neurotransmitter. Inspection of the GENSAT BAC-EGFP lines identified a number of genes with expression restricted within the cerebral cortex to distinct layers. Two such genes, Etv1, encoding the transcription factor etv1 (an ETS-binding transcription factor, also known as ER81), and the neurotensin 1 receptor (Ntsr 1 ), have been shown in BAC-EGFP transgenic lines to be localized respectively in layers 5 and 6 of the neocortex. There is a similar situation in the striatum, where all of the main subtypes of projection neurons use GABA as a neurotransmitter. In contrast with the cortex, striatal neuron projection subtypes are intermingled with each other. However, the two main subtypes, striatonigral and striatopallidal projection neurons, selectively express the Drd 1a and Drd2 dopamine receptors, respectively (Gerfen et al., 1990). Thus, these genes provide good candidates to target Cre expression to specific forebrain circuits. Figure 2 shows the Cre-induced EGFP expression in BAC-Cre transgenic lines produced with these four genes. Directed Cre expression is produced in different BAC-Cre transgenic lines in layer 5 corticostriatal neurons (Etv1 BAC-Cre), in layer 6 corticothalamic neurons (Ntsr1 BAC-Cre), in striatopallidal neurons (Drd2 BAC-Cre), and in striatonigral neurons (Drd1 BAC-Cre). The robustness of Cre expression in these lines is such that the EGFP-immunohistochemical labeling produced with the EGFP reporter line clearly shows the specific axonal projections in each of these Cre driver lines.

The Etv1 and Ntsr1 BAC-Cre lines display expression that is restricted principally to the cerebral cortex, and within the cortex to neocortical areas and layers 5 and 6, respectively. In contrast, the Drd1a and Drd2 BAC-Cre lines, while producing specific expression in striatonigral and striatopallidal neurons, also produce expression in other brain regions. Such non- 
Pcp2 BAC-Cre

A

Cmtm5 BAC-Cre

oligodendrocyte protein

B

\section{Slc6a4 BAC-Cre ER}

\section{tamoxifen- inducible Cre}

\section{C}

Figure 3. $A, A P c p 2 B A C-C r e$ transgenic line produces Cre expression that is restricted to the cerebellum localized to Purkinje cell neurons as seen in a sagittal brain section. $B, A C m t m 5$ BAC-Cre transgenic line produces Cre expression in oligodendrocyte cells throughout the brain. $\mathbf{B i}$, Labeled cells in a forebrain area including the cerebral cortex, underlying white matter, and striatum are seen to have the morphology of oligodendrocytes. C, Inducible Cre expression occurs in a serotonin transporter (SIca4) BAC-ER-Cre transgenic line. Mice treated with saline show no Cre expression, whereas a single treatment with tamoxifen (6 mg/kg, i.p.) produces Cre expression in serotonin neurons in the dorsal raphe.

striatal expression may provide some limitation for certain experiments compared with lines in which expression is restricted to striatal neurons. Another Drd2 BACCre line (ER43) does have expression restricted primarily to striatopallidal neurons, with very little in other brain regions (supplemental Fig. 6, available at www.

jneurosci.org as supplemental material). As with the partial expression in the Chat BAC-Cre line (GM60), the more restricted expression is most likely attributable to fewer copies of the BAC-Cre construct being inserted in the ER43 line than in the ER44 line. Again, the evidence for this is somewhat empirical in that it appears that there are differences in the level of Drd2 Cre-induced EGFP in different brain regions based on the robustness of labeling in axonal projections. A comparison of the Cre-induced EGFP labeling be- tween these two lines shows an overall reduction in the ER43 line, which is likely caused by a dose effect. In this case, such reduced expression may provide for more selective targeting of striatopallidal neurons, compared with the line in which expression is more widespread.

Purkinje cell protein (Pcp2) and oligodendrocyte protein ( $\mathrm{Cmtm5})$ BAC-Cre transgenic lines

A major advantage of the use of BAC constructs to direct recombinase expression to specific neuron types is the general applicability to a large variety of gene loci. To further test this approach, two other genes expressed in either a specific neuron type, the Purkinje cell protein 2 ( $P c p 2)$ expressed specifically in the cerebellum by Purkinje cells and the CKLF-like MARVEL transmembrane dopamine-containing 5 protein $(\mathrm{Cmtm} 5)$ expressed in oligodendrocytes were used to generate BACCre driver lines. As shown in Figure 3, these lines produced specific Cre expression in the appropriate brain cell types.

Serotonin transporter (Slc6a4) BAC-ER inducible Cre transgenic lines

To further enhance the targeting of Cre expression to specific neuron types using BAC-Cre constructs, a modification was introduced to provide for Cre expression to be induced by treatment of animals with tamoxifen. This construct was used to produce an Slca4 BAC-ER-Cre transgenic line, which was crossed with the ROSA26-EGFP mice. Offspring, which were at least $60 \mathrm{~d}$ of age, were treated with either vehicle or tamoxifen for 1-4 d and allowed to survive for 1 additional week. The brains of tamoxifen-treated animals displayed a number of EGFP-positive neurons in the dorsal raphe, whereas vehicle-treated animals did not show any labeled neurons (Fig. 3).

\section{Discussion}

Results presented demonstrate the general applicability of using BAC constructs for efficient production of Cre drivers targeting specific cell populations in the brain, including both neuronal and glial cell types. This extends the approach of the GENSAT project, in which transgenic mouse lines have been produced with EGFP expressed in neuron populations using BAC constructs containing genes expressed in the brain (Gong et al., 2003). Over 500 BAC-EGFP transgenic mouse lines have been characterized, in which EGFP is expressed in select neuronal and glial cell populations expressing the endogenous cognate gene. These BAC-EGFP transgenic lines provide a valuable resource by enabling specific neurons types to be identified (for example, in neurophysiologic studies) (Day et al., 2006). Substitution of Cre for EGFP adds functional utility for neurobiologic studies, allowing gene function to be altered in specific neuron or glial cell populations to enable a variety of different studies.

Experience gained with BAC-EGFP transgenic lines indicates that the most important determinant for correct expression from BAC vectors is inclusion of as much of the intergenic region surrounding a gene of interest within the BAC construct as possible. Nonetheless, it has proven generally necessary to screen 
multiple transgenic founder lines generated with the same BAC-EGFP construct to identify lines that produce expression patterns matching that of the endogenous gene. Both incomplete and ectopic expression is observed regularly using BAC transgenic vectors. However, it has been our experience that evaluation of the three or four founder lines produced in a single session of pronuclear injection will result in identification of an accurately expressing line for $\sim 85 \%$ of the vectors, provided that the transcription unit chosen gene is less than $\sim 150 \mathrm{~kb}$ and the BAC DNA is prepared properly (GENSAT, unpublished data). Screening lines generated with BAC-Cre constructs produced similar results, with the added complication that lines expressing constitutive Cre expression often reflect the cumulative expression history of the gene throughout the organism. Two examples of this type of expression were observed with the Slca4 and Th BAC-Cre constructs, in which Cre expression was present in the adult in both neurons expressing these genes in the adult and in other neurons, in which these genes are expressed transiently during development. In the case of the Slca4 BAC-Cre constructs, other lines showed expression restricted to neurons expressing the gene in the adult. On the other hand, of the Th BAC-Cre lines analyzed, none produced expression restricted only to neurons that express $T h$ in the adult. In these lines, expression of Cre in neurons that do not express $T h$ in the adult corresponds to neurons that have been reported to express $T h$ transiently during development. In attempting to direct Cre expression to neurons in which Th is expressed in the adult, BACs containing other genes, such as DAT (dopamine transporter), might be more appropriate. For some experiments, it may be useful to be able to target Cre to neurons that only transiently express the gene of interest during development. In such cases, it is important to take into consideration that the effects of recombination occur only during the developmental period during which the gene is transiently expressed. Thus, injection of vectors incorporating a "floxed" construct into the adult brain would not result in recombination in neurons that had only transiently expressed Cre during development.

In other BAC-Cre transgenic lines, expression of Cre is restricted to a subset of neurons expressing the endogenous gene. Two such transgenic lines are described in this study, including Chat and Drd2 BACCre transgenic lines. The most likely cause of the incomplete expression of Cre in these lines is differences in the number of copies of the BAC-Cre construct that are incorporated in the transgenic line. Such copy number effects are likely attributable to the fact that Cre activity will vary depending on the relative expression of the gene in specific cell types targeted. In BAC-Cre lines with lower copy numbers, the Cre-induced EGFP labeling may appear to be generally reduced throughout the brain. As seen in the two cases described, such reduced expression does not produce different levels of expression in a particular subset of neurons, but rather produces the absence of detectable expression in one subset while retaining expression in other sets of neurons. However, it is important to understand that such partial patterns of expression are a consequence of the efficiency of recombination produced by the reporter line and will have to be determined for other floxed lines on a case-by-case basis. Nonetheless, such reduced Cre expression may be quite useful for targeting effective Cre recombination to specific subsets of neurons in vivo.

The utility of transgenic mouse lines with Cre expression directed to specific neuron populations will depend on the availability of transgenic lines engineered with lox sites positioned to alter expression of specific genes. Several types of such transgenic lines have been developed. In one type, lox sites are inserted to remove all or portions of the coding sequence of endogenous genes, which when crossed with a BAC-Cre transgenic line will remove specific gene products from neurons expressing Cre. In another type, gene constructs are used to drive exogenous gene products using lox sites flanking a stop codon to release promoter-driven expression. For example, such a construct is used to express wheat germ agglutinin in neurons for the purpose of tracing the transsynaptic connections of neurons expressing Cre (Braz et al., 2002). In this study, a transgenic line in which lox sites were inserted to flank a stop codon in front of the Rosa26 promoter (Soriano, 1999), followed by the coding sequence for EGFP, was used to determine the efficacy of Cre expression in specific brain cell populations. This reporter line provides a means of determining that Cre expression is present in particular neuron or glial cell types. However, the efficiency of recombinase activity to remove floxed genes may vary in different transgenic constructs. Consequently, whether such expression effects changes in gene expression of other floxed gene constructs must be verified on a case-by-case basis.

The present study establishes the general utility of using BAC-Cre constructs to produce transgenic mouse lines in which Cre recombinase expression is directed to specific neuron and glial cell types within the brain. We conclude that generation of cell-specific and inducible Cre driver lines can be efficient using engineered BAC vectors and that in some cases, this strategy could provide important advantages over knock-in strategies for production of recombinase driver lines. The 14 lines described here will be added to the GENSAT BAC-EGFP lines to be distributed through the Mutant Mouse Regional Resource Center (MMRRC; http://www. mmrrc.org) at the University of California, Davis. Characterization of the patterns of Cre expression for these lines will be provided on the GENSAT website, which will include data from adult brain sections and from developmental patterns as appropriate. Moreover, as part of the GENSAT project, $>25$ new BAC-Cre driver lines will be added annually to those available through the MMRRC. Selection of new BAC-Cre driver lines will be made from the expanding number of BAC-EGFP transgenic lines produced by the GENSAT project. The goal of the GENSAT project is to provide a repository of BAC-Cre transgenic lines targeted to diverse but very specific neuron and glial brain cell types to facilitate neuroscience research.

\section{References}

Aller MI, Jones A, Merlo D, Paterlini M, Meyer AH, Amtmann U, Brickley S, Jolin HE, McKenzie AN, Monyer H, Farrant M, Wisden W (2003) Cerebellar granule cell Cre recombinase expression. Genesis 36:97-103.

Branda CS, Dymecki SM (2004) Talking about a revolution: the impact of site-specific recombinases on genetic analyses in mice. Dev Cell 6:7-28.

Braz JM, Rico B, Basbaum AI (2002) Transneuronal tracing of diverse CNS circuits by Cremediated induction of wheat germ agglutinin in transgenic mice. Proc Natl Acad Sci USA 99:15148-15153.

Day M, Wang Z, Ding J, An X, Ingham CA, Shering AF, Wokosin D, Ilijic E, Sun Z, Sampson AR, Mugnaini E, Deutch AY, Sesack SR, Arbuthnott GW, Surmeier DJ (2006) Selective elimination of glutamatergic synapses on striatopallidal neurons in Parkinson disease models. Nat Neurosci 9:251-259.

Gerfen CR, Engber TM, Mahan LC, Susel Z, Chase TN, Monsma FJ, Sibley DR (1990) D1 and $\mathrm{D} 2$ dopamine receptor regulated gene expression of striatonigral and striatopallidal neurons. Science 250:1429-1432.

Gong S, Yang XW, Li C, Heintz N (2002) Highly efficient modification of bacterial artificial 
chromosomes (BACs) using novel shuttle vectors containing the R6Kgamma origin of replication. Genome Res 12:1992-1998.

Gong S, Zheng C, Doughty ML, Losos K, Didkovsky N, Schambra UB, Nowak NJ, Joyner A, Leblanc G, Hatten ME, Heintz N (2003) A gene expression atlas of the central nervous system based on bacterial artificial chromosomes. Nature 425:917-925.

Gu H, Marth JD, Orban PC, Mossmann H, Rajewsky K (1994) Deletion of a DNA polymerase beta gene segment in T cells using cell typespecific gene targeting. Science 265:103-106.

Heintz N (2001) BAC to the future: the use of bac transgenic mice for neuroscience research. Nat Rev Neurosci 2:861-870.

Indra $\mathrm{AK}$, Warot X, Brocard J, Bornert JM, Xiao JH, Chambon P, Metzger D (1999) Temporally-controlled site-specific mutagenesis in the basal layer of the epidermis: comparison of the recombinase activity of the tamoxifen-inducible Cre-ER(T) and CreER(T2) recombinases. Nucleic Acids Res 27:4324-4327.

Komori K, Sakai M, Karasawa N, Yamada K, Nagatsu I (1991) Evidence for transient expression of tyrosine hydroxylase immunoreactivity in the mouse striatum and the effects of colchicine. Acta Histochem Cytochem 24:223-231.

Kuhn R, Torres RM (2002) Cre/loxP recombination system and gene targeting. Methods Mol Biol 180:175-204.

Kuhn R, Schwenk F, Aguet M, Rajewsky K (1995) Inducible gene targeting in mice. Science 269:1427-1429.

Lakso M, Sauer B, Mosinger Jr B, Lee EJ, Manning RW, Yu SH, Mulder KL, Westphal H (1992) Targeted oncogene activation by site-specific recombination in transgenic mice. Proc Natl Acad Sci USA 89:6232-6236.

Lebrand C, Cases O, Wehrle R, Blakely RD, Edwards RH, Gaspar P (1998) Transient developmental expression of monoamine transporters in the rodent forebrain. J Comp Neurol 401:506-524.

Lindeberg J, Usoskin D, Bengtsson H, Gustafsson A, Kylberg A, Söderström S, Ebendal T (2004) Transgenic expression of Cre recombinase from the tyrosine hydroxylase locus. Genesis 40:67-73.

Marin F, Herrero MT, Vyas S, Puelles L (2005) Ontogeny of tyrosine hydroxylase mRNA expression in mid- and forebrain: neuromeric pattern and novel positive regions. Dev Dyn 234:709-717.

Scott MM, Wylie CJ, Lerch JK, Murphy R, Lobur
K, Herlitze S, Jiang W, Conlon RA, Strowbridge BW, Deneris ES (2005) A genetic approach to access serotonin neurons for in vivo and in vitro studies. Proc Natl Acad Sci USA 102:16472-16477.

Soriano P (1999) Generalized lacZ expression with the ROSA26 Cre reporter strain. Nat Genet 21:70-71.

Tsien JZ, Chen DF, Gerber D, Tom C, Mercer EH, Anderson DJ, Mayford M, Kandel ER, Tonegawa S (1996) Subregion- and cell type-restricted gene knockout in mouse brain. Cell 87:1317-1326.

Yang XW, Model P, Heintz N (1997) Homologous recombination based modification in Escherichia coli and germline transmission in transgenic mice of a bacterial artificial chromosome. Nat Biotechnol 15:859-865.

Zhuang X, Masson J, Gingrich JA, Rayport S, Hen R (2005) Targeted gene expression in dopamine and serotonin neurons of the mouse brain. J Neurosci Methods 143:27-32.

Zinyk DL, Mercer EH, Harris E, Anderson DJ, Joyner AL (1998) Fate mapping of the mouse midbrain-hindbrain constriction using a site-specific recombination system. Curr Biol 8:665-668. 